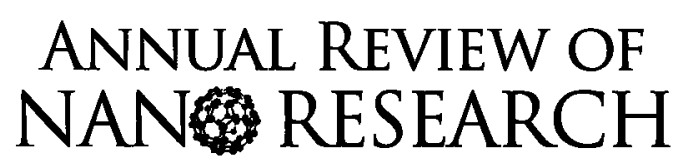

VOLUME 1 


\title{
ANNUAL REVIEW OF NANO RESEARCH
}

Series Editors: Guozhong Cao (University of Washington, USA)

C Jeffrey Brinker (University of New Mexico \& Sandia National Laboratories, USA)

\author{
Vol. 1: ISBN-13 978-981-270-564-8 \\ ISBN-10 981-270-564-3 \\ ISBN-13 978-981-270-600-3 (pbk) \\ ISBN-10 981-270-600-3 (pbk)
}




\section{ANNUAL REVIEW OF NANG RESEARCH}

VOIUM: 1

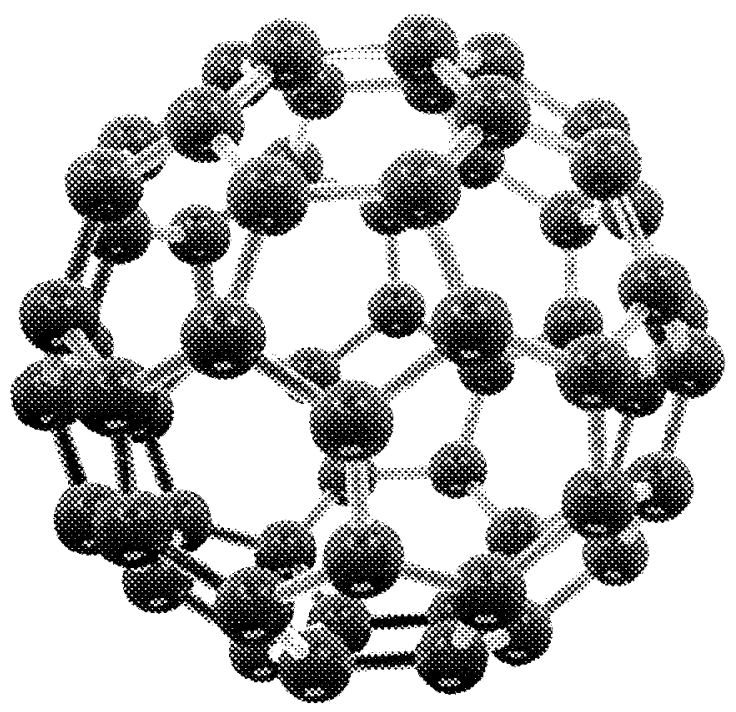

EDITORS

GUOZHONG CAO

University of Washington, USA

C. JEFFREY BRINKER

University of New Mexico and Sandia National Laboratories, USA

\section{Now world Scientific}




\section{Published by}

World Scientific Publishing Co. Pte. Ltd.

5 Toh Tuck Link, Singapore 596224

USA office: 27 Warren Street, Suite 401-402, Hackensack, NJ 07601

UK office: 57 Shelton Street, Covent Garden, London WC2H 9HE

\section{British Library Cataloguing-in-Publication Data}

A catalogue record for this book is available from the British Library.

\section{ANNUAL REVIEW OF NANO RESEARCH, Vol. 1}

Copyright $₫ 2006$ by World Scientific Publishing Co. Pte. Ltd.

All rights reserved. This book, or parts thereof, may not be reproduced in any form or by any means, electronic or mechanical, including photocopying, recording or any information storage and retrieval system now known or to be invented, without written permission from the Publisher.

For photocopying of material in this volume, please pay a copying fee through the Copyright Clearance Center, Inc., 222 Rosewood Drive, Danvers, MA 01923, USA. In this case permission to photocopy is not required from the publisher.

ISBN-13 978-981-270-564-8

ISBN-10 981-270-564-3

ISBN-13 978-981-270-600-3 (pbk)

ISBN-10 981-270-600-3 (pbk) 


\section{TABLE OF CONTENTS}

Preface

Contributing Authors

$x v$

$x v i i$

Chapter 1. Recent Progress in Syntheses and Applications of Inverse Opals and Related Macroporous Materials Prepared by Colloidal Crystal Templating

Justin C. Lytle and Andreas Stein

1. General Introduction

2. Synthesis of Colloidal Crystals

2.1. Synthesis of 3DOM Structures

2.1.1. Synthesis of Simple Oxides

2.1.2. Synthesis of Ternary Oxides and Higher Compositions

2.1.3. Synthesis of Non-Oxides

2.1.4. Synthesis of Metals

2.1.5. Synthesis of Semiconductors

2.1.6. Synthesis of Polymers

2.1.7. Synthesis of Hydrogels

2.1.8. Synthesis of Hybrid Compositions and Composites

2.1.9. Nanocasting with 3DOM Templates 21

2.1.10. Hierarchical Structuring 22

2.1.11. Two-Dimensional Pore Arrays 28

3. Properties and Applications of 3DOM Materials 29

3.1. Mechanical Characterization 29

3.2. Optical Applications $\quad 30$

3.2.1. Photonic Crystals $\quad 30$

3.2.2. Modification of Spontaneous Emission 33

3.2.3. Tunable Photonic Crystals 35

3.2.4. Metallic and Metallodielectric Photonic Crystals via Colloidal Crystal Templating $\quad 38$

3.2.5. Defects and Deformations in Photonic Crystals

3.2.6. 3DOM Pigments 
3.2.7. Dye-Sensitized Titania Photonic Crystals 42

3.2.8. Surface-Enhanced Raman Spectroscopy 44

3.3. Sensors 44

3.3.1. Response Based on Changes in Refractive Index 45

3.3.2. Response Based on Changes in Pore Spacing or Pore Geometry 46

3.3.3. Response Based on Changes in Surface Electronic States 47

3.3.4. Electrochemical Response $\quad 48$

3.4. Magnetic Properties 49

3.5. Catalysis 51

3.6. Electrode and Battery Applications 54

3.7. Sorption and Wetting Behavior $\quad 57$

3.8. Bioactive Materials $\quad 60$

3.9. Pseudomorphic Transformation of 3DOM Materials 62

4. Conclusion 64

Acknowledgements $\quad 64$

References 64

Chapter 2. Photonic Crystals: Fundamentals and Applications 81 Álvaro Blanco and Cefe López

1. Introduction 81

1.1. Photonic Band Gap Materials $\quad 83$

1.2. Optical Characterization 91

1.2.1. Photonic Bands Interpretation 93

$\begin{array}{lr}\text { 1.3. Applications } & 95\end{array}$

1.4. Metamaterials 100

2. Preparation of Photonic Crystals 102

2.1. One-Dimensional Systems 102

2.2. Two-Dimensional Systems 104

2.3. Three-Dimensional Systems 107

2.3.1. Colloidal Crystals 111

2.3.2. Bare Opals 112

2.3.3. Further Processing 121

2.3.4. Composites 123

3. Summary 


\section{Chapter 3. Nanoparticle-Micelle: A New Building Block for} Facile Self-Assembly and Integration of 2-, 3Dimensional Functional Nanostructures

Hongyou Fan and C. Jeffrey Brinker

1. Introduction

2. Synthesis of NP-Micelles 155

3. Synthesis of Ordered NP Arrays 165

3.1. Synthesis of Hierarchically Ordered Mesostructured NP Arrays

3.2. Synthesis of Ordered NP Arrays in Thin Films 172

4. Integration of NP Arrays for Charge Transport Study 180

5. Conclusions and Outlook

Acknowledgements

\section{Chapter 4. Electrospinning Nanofibers with Controlled} Structures and Complex Architectures

Dan Li, Jesse T. McCann, Manual Marquez, and Younan Xia

1. Introduction

2. Experimental Setup for Electrospinning

3. History and Mechanism of Electrospinning

4. Nanofibers Containing Nanoscale Fillers

4.1. Nanoparticles as the Fillers

4.2. Nanowires and Nanotubes as the Fillers

4.3. Nanosheets as the Fillers

5. Electrospinning with a Dual-Capillary Spinneret

5.1. Core/Sheath Nanofibers

5.2. Hollow Nanofibers with Controlled Surface Structures 201

5.3. Improvement of Electrospinnability 203

6. Porous Nanofibers

6.1. Porous Nanofibers by Bicomponent Spinning 
6.2. Porous Fibers by Polymer-Solvent Phase Separation 205

7. Complex Nanofibers via Post-Spinning Treatment 207

8. Ordered Architectures of Electrospun Nanofibers 209

9. Concluding Remarks 212

Acknowledgements 212

References $\quad 212$

Chapter 5. Structure of Doped Single Wall Carbon Nanotubes 215 L. Duclaux, J.-L. Bantignies, L. Alvarez, R. Almairac, and J.-L. Sauvajol

1. Introduction

2. Structure of Doped SWCNTs (X-Ray Diffraction and Neutron Diffraction Studies)

2.1. Electron Acceptors

2.2. Electron Donors

2.2.1. Insertion of $\mathrm{Li}$ and $\mathrm{Na}$

224

2.2.2. Heavy Alkali Metals (K, Rb, Cs) 226

3. The Local Structure (EXAFS and TEM) 233

3.1. Rubidium Doping 234

3.2. Iodine Doping 238

4. Raman Spectroscopy of Bundled SWCNT 241

4.1. Raman Spectra of Alkali-Doped SWCNT Bundles 243

4.1.1. Doping at Saturation Level 243

4.1.2. Progressive Doping 244

5. Conclusion 247

References 250

Chapter 6. Electron Transport in Molecular Electronic Devices 255 Shimin Hou, Zekan Qian, and Rui Li

1. Introduction 255

2. Experimental Progress in Molecular Electronic Devices 256

3. The NEGF+DFT Approach 265

3.1. Current Formula for an Electrode-Molecule-Electrode Junction

3.2. Implementation of the NEGF+DFT Approach 
3.2.1. Green's Function Part: Calculating the Density Matrix in an Open System

3.2.2. DFT Part: Calculating the KS Hamiltonian Matrix from the Density Matrix

3.2.3. Achieving Self-Consistency

3.3. Application and Challenge of the NEGF+DFT Approach

References

\section{Chapter 7. Structure, Properties, and Opportunities of Block} Copolymer/Particle Nanocomposites

Lindsay Bombalski, Jessica Listak, and Michael R. Bockstaller

1. Introduction

2. Structure Formation in BCP Hybrid Materials - Theory and Simulation

3. Structure Formation of BCP Hybride Materials - Experiments 306

3.1. Equilibrium BCP/Particle Composite Morphologies

3.2. Nonequilibrium BCP/Particle Composite Morphologies

4. Structure-Property Relations and Applications of BCP/NP Hybrid Materials

4.1. Properties Capitalizing on Effective Properties of Randomized NP Inclusions

4.2. Properties Capitalizing on Cooperative Phenomena of Discrete Particle Arrangements

5. Conclusion

Acknowledgements

References

Chapter 8. Electro-Oxidation and Local Probe Oxidation of Nano-Patterned Organic Monolayers

Daan Wouters and Ulrich S. Schubert

1. Introduction

2. Monolayer Formation 
2.1. Thiolate Monolayers 341

2.2. Alkylsilane Monolayers $\quad 342$

3. Monolayer Patterning 347

3.1. Monolayer Patterning by Means of Energetic Beams $\quad 350$

3.2. Monolayer Patterning by Means of Local Probes 357

3.2.1. Local Probe Oxidation 357

3.2.2. Local Probe Electro-Oxidation of SAMs 361

3.2.3. Other Examples of Local Probe ElectroOxidation

4. Summary

Acknowledgements $\quad 375$

References

Chapter 9. Recent Development of Organogels Towards Smart and Soft Materials

385

Norifumi Fujita, Pritam Mukhopadhyay, and

Seiji Shinkai

1. General Introduction

2. First Generation Organogels 386

2.1. Steroid-Based Gelators 387

2.2. Anthracene-Based Gelators 387

2.3. Amino Acid and Ammonium Carbamate-Based Gelators

2.4. Sugar-Based Organogels 391

2.5. Chiral Gelators 391

2.6. Glycoluril-Based and Macrocycle-Based Gelators 392

2.7. Gelators Based on Complex Building Blocks 393

3. Second Generation Organogels 394

3.1. Host-Guest Interaction 395

3.2. H-Bonding Interaction 399

3.3. Donor-Acceptor Interaction 406

3.4. Metal-Responsive Organogels 408

3.5. Gels with Novel Optical Properties 412

3.6. Photo-Responsive Organogels 416

3.7. Redox Active Organogels 419

3.8. Light Harvesting Organogel Systems 420

4. Miscellaneous Organogels 423 
5. Biomedical Applications 424

6. Conclusions and Future Outlook 425

$\begin{array}{ll}\text { References } & 425\end{array}$

Chapter 10. Biosensors Based on Gold Nanoparticle Labeling 429 Robert Möller and Wolfgang Fritzsche

1. Introduction

2. General Features of Gold Nanoparticles: Synthesis and Bioconjugation

3. Detection of Gold Nanoparticles - DNA Conjugates 434

3.1. Optical Detection 434

3.1.1. Homogeneous Detection 434

3.1.2. Heterogeneous Detection 438

3.1.3. Optical Scattering 441

3.1.4. Raman Scattering 443

3.1.5. Surface Plasmon Resonance (SPR) Imaging 446

3.1.6. Photothermal Imaging 448

3.2. Micromechanical Detection 449

3.2.1. Quartz-Crystal Microbalances (QCM) 449

3.2.2. Microcantilever 450

3.3. Electrical Detection 451

3.4. Electrochemical Detection 451

3.5. Resistive or Capacitive Detection 453

4. Further Applications of Gold Nanoparticles for Biosensing 455

5. Outlook 458

References $\quad 459$

Chapter 11. Quantum Dot Applications in Biotechnology:

Progress and Challenges

467

Cheng-An J. Lin, Jimmy K. Li, Ralph A. Sperling, Liberato Manna, Wolfgang J. Parak, and Walter H. Chang

1. Introduction

2. Quantum Dots: Synthesis and Surface Modification for Their Use in Biomedical Research 
2.1. Synthesis of Colloidal Semiconductor Nanocrystals $\quad 470$

2.2. Hydrophilic Modification 473

2.2.1. Ligand Exchange $\quad 474$

2.2.2. Surface Silanization 476

2.2.3. Amphiphilic Polymer/Surfactant Coating $\quad 477$

2.2.4. Conclusions 480

2.3. Bioconjugate Techniques 480

2.4. Synthesis of 'Greener' Quantum Dots (GQDs) 483

3. Properties of Quantum Dots 487

3.1. Some Basic Photo-Physical Properties 487

3.2. Cytotoxicity/Biocompatibility 491

4. Quantum Dots as a Cellular Probe 493

4.1. Labeling of Cellular Structures and Receptors 493

4.2. Incorporation of Quantum Dots by Living Cells 495

4.3. Tracking the Path and the Fate of Individual Cells with Quantum Dot Labels $\quad 499$

5. Quantum Dots as a Biosensors 502

5.1. Quantum Dots as FRET Donor 502

5.2. Quantum Dots as FRET Acceptor 505

6. Quantum Dots as in vivo Probes 506

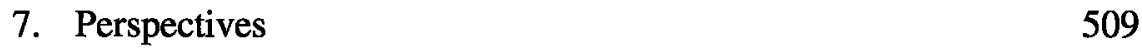

Acknowledgements $\quad 510$

$\begin{array}{ll}\text { References } & 510\end{array}$

Chapter 12. DNA-Based Artificial Nanostructures 531

Giampaolo Zuccheri, Marco Brucale,

Alessandra Vinelli, and Bruno Samori

1. Introduction 531

2. Affinity vs. Specificity in DNA Interactions 532

3. Structural Codes for DNA in the Nanoscale: Shape and Dynamics

3.1. The DNA Shape Code: How Local Deformations Can Affect the Average Molecular Shape

3.2. DNA Flexibility: Curvature is Only Half of the Story (but the Story is not Complete Yet)

3.3. Surface-DNA Interactions can be Sequence-Dependent 538 
4. A Practical Application of the Watson-Crick DNA Code: DNA Chips and DNA Detection

5. Base-Pairing for Nanoscience and Nanotechnology

5.1. An Evolving Fauna of DNA-Based Molecular Nanostructures

5.2. Hybrid Nanostructures Based on DNA Assembly: Metal Nanoparticles Plus DNA as an Example

5.3. Nature and Nanotechnology are a Matter of Hierarchy (and Topology)

5.3.1. Zero-Dimensional Topologies in DNA Artificial Nanostructures: Discrete DNA Constructs

5.3.2. Mono-Dimensional Topologies: Linear Arrays of Supramolecularly Connected Components to Make DNA Nano-Objects

5.3.3. Two-Dimensional Topologies of DNA Tiles

5.4. Raising the Size and Complexity: Algorithmic Assembly, DNA Origami, and Other Assemblies on Long Template Strands

5.5. Building 3D Objects

5.6. Strategies to Enhance the Structural Rigidity of the Nanostructures

5.7. The Enhancement of Symmetry in the Assembly: An Alternative Strategy

5.8. The Temporal Dimensionality

6. Conclusions and Outlook

\section{Chapter 13. Recent Progress on Bio-Inspired Surface with Special Wettability Shutao Wang, Huan Liu, and Lei Jiang}

1. Introduction

2. Some Basic Aspects about Surface Wettability 575

2.1. Hydrophilicity and Hydrophobicity

2.2. Wenzel's Model and Cassie's Model

2.3. Superhydrophilicity and Superhydrophobicity

3. Unique Superhydrophobic Surfaces in Nature 
4. Artificial Superhydrophobic Surface

4.1. Towards the Simple Process

4.2. Towards Environmental Stability

4.3. Towards Multi-Function

591

5. Superhydrophilic Surfaces

6. Surfaces with Tunable Wettability from Superhydrophobic to Superhydrophilic

602

7. Responsive Surfaces Between Superhydrophobicity and Superhydrophilicity

606

7.1. Single Stimuli-Responsive Surfaces

606

7.1.1. Photo-Responsive Surfaces

606

7.1.2. pH-Responsive Surfaces

611

7.1.3. Thermal-Responsive Surfaces

612

7.1.4. Electric-Field Responsive Surfaces

614

7.1.5. Mechanical Force Responsive Surfaces

616

7.2. Multi Stimuli-Responsive Surfaces

618

8. Conclusions and Outlook

621

References 


\section{PREFACE}

Nanotechnology is so pervasive; it has an impact on all research fields. Scientists, engineers, pharmacists, and physicians from all different disciplines, and all branches of physical and life sciences and engineering, are either actively contributing to the rapid progress of the nanotechnology field, or will soon be affected by evolving nanotechnology. There are many archive journals and monographs dedicated to publishing the most recent research progress in nanotechnology with varied focus. Many well-established journals in more traditional disciplines have an increasing coverage of nanotechnology research. Not only are the specialty journals making increasing efforts to keep up with the rapid advancement of nanotechnology, but the popular science journals and books are also keeping up the pace to inform the general population and policymakers. More and more symposia, conferences, and workshops focusing on nanotechnology have been organized. Similarly, more and more short courses and regular courses on nanotechnology are offered to educate both graduate and undergraduate students.

In spite of the broad impact and evolving development of nanotechnology, it is most unlikely its own industry will form. Instead, it penetrates into every sector of industry and technology, and all aspects of mankind's activity. Consequently, there is a constant flux of emerging new research directions in nanotechnology. It becomes obvious that nanotechnologists face great difficulty in keeping themselves embraced with the most recent development in the nanotechnology field. Yet many developments and understanding achieved in different focal areas can be directly applicable to other sub-fields of nanotechnology. Review articles by authority experts in their own sub-fields play a significant role in summarizing the recent development in selected topic areas, and thus serve two vital purposes: (1) to present a comprehensive and coherent description of the state-of-the-art understanding by distilling the most valuable experimental results and theories from otherwise segmented and scattered literature, and (2) to offer critical opinions regarding the challenges, promises, and possible future developing directions. Review 
articles are published in most archive journals, and are thus associated with the focal areas that journals are devoted to. This annual review collects excellent review articles authored by the authority experts and their colleagues in various sub-fields of nanotechnology and thus offer easy access of critical opinions of the most recent developments in nanotechnology.

This volume of Annual Review of Nano Research includes 13 review articles and offers a concise review detailing recent advancements in a few selected topics in nanotechnology. Topics in this volume consist of inverse opals and macroporous materials, photonic crystals, and molecular electronics. Other areas involving functional nanostructures, oxide nanofibers, carbon nanotubes, and nanocomposites are also covered in great detail. In addition, many different areas related to organic materials, nano-patterned materials, and biological materials are also described extensively.

Dr. Tammy P. Chou has put in many hours and has placed great effort in editing and formatting the review articles published in this volume. Mr. Yeow-Hwa Quek from World Scientific Publishing coordinated a lot of effort to make the publication of this volume possible.

Guozhong Cao

Seattle, WA

C. Jeffrey Brinker

Albuquerque, NM 


\section{CONTRIBUTING AUTHORS}

Almairac, R.

* Université Montpellier II, France

Alvarez, L.

* Université Montpellier II, France

Bantignies, J-L.

* Université Montpellier II, France

Blanco, Álvaro

* Instituto de Ciencia de Materiales de Madrid (CSIC), Spain

* Unidad Asociada CSIC-U, Spain

Bockstaller, Michael R.

* Carnegie Mellon University, USA

Bombalski, Lindsay

* Carnegie Mellon University, USA

Brinker, C. Jeffrey

* Sandia National Laboratories, USA

* University of New Mexico, USA

Brucale, Marco

* University of Bologna Via Irnerio, Italy

Chang, Walter $\mathrm{H}$.

* Chung Yuan Christian University, Taiwan

Duclaux, L.

* Polytech'Savoie, Université de Savoie, France

Fan, Hongyou

* Sandia National Laboratories, USA

* University of New Mexico, USA 
Fritzsche, Wolfgang

*Institute for Physical High Technology, Germany

Fujita, Norifumi

* Kyushu University, Japan

Hou, Shimin

* Peking University, China

Jiang, Lei

* Chinese Academy of Sciences, China

* National Center for NanoScience and Technology, China

Li, Dan

* University of Washington, USA

* Philip Morris, USA

Li, Jimmy $K$.

* Chung Yuan Christian University, Taiwan

Li, Rui

* Peking University, China

Lin, Cheng-An J.

* Ludwig Maximilians University, Germany

* Chung Yuan Christian University, Taiwan

Listak, Jessica

* Carnegie Mellon University, USA

Liu, Huan

* National Center for NanoScience and Technology, China

López, Cefe

* Instituto de Ciencia de Materiales de Madrid (CSIC), Spain

* Unidad Asociada CSIC-U, Spain

Lytle, Justin C.

* University of Minnesota, USA 
Manna, Liberato

* Universita delgi studi Lecce, Italy

Marquez, Manual

* Center for Theoretical and Computational Nanosciences, USA

* Arizona State University, USA

* Philip Morris, USA

McCann, Jesse T.

* University of Washington, USA

Möller, Robert

* Friedrich-Schiller-University, Germany

Mukhopadhyay, Pritam

* Kyushu University, Japan

Parak, Wolfgang J.

* Ludwig Maximilians University, Germany

Qian, Zekan

* Peking University, China

Samorì, Bruno

* University of Bologna Via Irnerio, Italy

Sauvajol, J-L.

* Université Montpellier II, France

Schubert, Ulrich S.

* Eindhoven University of Technology, Netherlands

* Ludwig Maximilians University, Germany

Shinkai, Seiji

* Kyushu University, Japan

Sperling, Ralph A.

* Ludwig Maximilians University, Germany 
Stein, Andreas

* University of Minnesota, USA

Vinelli, Alessandra

* University of Bologna Via Irnerio, Italy

Wang, Shutao

* Chinese Academy of Sciences, China

Wouters, Daan

* Eindhoven University of Technology, Netherlands

Xia, Younan

* University of Washington, USA

Zuccheri, Giampaolo

* University of Bologna Via Irnerio, Italy 